\title{
A Common Frame for Formal Imagination
}

\author{
(Author names omitted for blind review)
}

Received: date / Accepted: date

\begin{abstract}
In this paper, we review three influential theories of imagination in order to understand how the dynamics of imagination acts could be modeled using formal languages. While reviewing them, we notice that they are not detailed enough to account for all the mechanisms involved in creating and developing imaginary worlds. We claim those theories could be further refined into what we call the Common Frame for Imagination Acts, which defines a framework that can be used to study the dynamics of imagination using formal languages, and we support our claim by showing how the framework allows to model certain imagination acts step by step. Then, we introduce the Rhombus of Imagination, which is a visual tool that can be used in conjunction with our framework to study the procedural structure of different kinds of imagination acts and identify their dynamic properties.
\end{abstract}

Keywords Imagination · Dynamic imagination · Reality-oriented development · Imaginary worlds · Formal models

\section{Introduction and Motivations}

Imagining is something we do everyday in our lives and in a wide variety of ways. When planning our next move in a game of chess, when picturing how we could decorate our new room, or even when listening to a story-teller, our mind creates, develops and evaluates imaginary worlds aimed to guide our actions, update our beliefs, or entertain us. Imagination has received a great deal of attention by philosophers, cognitive scientists and psychologists, as it can be seen in works like Walton (1990), Currie and Ravenscroft (2002), Nichols (2006), or Kind (2016b). Its interest within the studies of the mind is beyond any doubt, and its relation to other mechanisms, such as emotions,

(Author affiliations omitted for blind review) 
behavior, desires and beliefs, makes imagination particularly interesting in many different areas.

There are many works in the literature that study the relation between imagination and other mental attitudes, such as knowledge (as in Kind and Kung (2016)), beliefs (in Sinhababu (2016)), desires (in Kind (2016a)), or how imagination affects our decision-making abilities (in Nanay (2016)). Some of these works also provide insights on how the mechanisms of creating and developing new imaginary worlds work. The aim of the present work is neither to study the outputs that result from an act of imagination, nor how acts of imagination affects our actions, or other mental attitudes. Instead, this work focuses on studying the dynamic processes involved in the act of creating and elaborating the imaginary worlds that result from such acts. In particular, we are interested in assessing how the existing theories behind the dynamics of imagination could be used as the basis for formal systems, such as a formal logic, or a computer simulation. Due to this, our main goal in this work will be to draw bridges between the intuitions captured in the reviewed theories of imagination and the main mechanics that could be represented using formal languages.

Many authors distinguish between different mental attitudes related to imagination. For instance, Funkhouser and Spaulding (2009) and Nichols and Stich (2000) distinguish "imagination" and "pretense" by requiring "pretense" to involve some behavior, or action. Other works like Balcerak-Jackson (2016) draw a distinction between "imagine", "suppose" and "conceive", which are seen as three different ways to refer to our ability to think about scenarios and objects that may or may not exist.

In this work we are interested in studying the dynamic processes involved in creating a so-called "imaginary world". There are a few important clarifications that should be kept in mind throughout the rest of the present paper, and which will help understand both the way we use certain terms, and the overall aim of our work:

1. When we talk about an "imaginary world", or an "imaginary scenario", we refer to any mental representation of a state of affairs that is not actual and, moreover, about which the relevant agent is aware of it not being actual. Therefore, representations of state of affairs used in supposition, or pretense, for instance, are also taken into account in our work, and we refer to them as imaginary worlds as well. Other kinds of mental representations, such as false beliefs, would not be the target of our model. This is because, in those cases, the agent is not aware of the fact that those mental representations do not correspond to the actual state of affairs.

2. Our main goal lies in understanding how those imaginary worlds are created and developed, but we do not consider, in our study, what happens when such worlds have been created, why the agent is creating them, or what outcomes follow from entertaining them ${ }^{1}$. Therefore, we do not re-

\footnotetext{
1 In relation to Van Leeuwen (2013), we can say that our interest lies in constructive imagining.
} 
strict ourselves to any particular kind of imagination act, like supposition, conception or pretense. As each and every one of these mental actions involve creating and elaborating representations of state of affairs that are not actual, we consider them all indistinctly. Therefore, we will be talking about "imagination" in a broad sense that includes those more specific kinds of imagination acts.

3. Furthermore, we focus on "voluntary" acts of imagination, which are those acts that are started by the agent consciously choosing an initial premise to characterize the imaginary scenario. As these are the kind of acts that are mainly explored in the theories we will examine, our work will also focus on them. Therefore, we will not consider involuntary imaginary scenarios such as dreams, hallucinations, or simply imaginings that pop up into the mind of the agent unexpectedly.

The content of the present paper is structured as follows. In Section 2, we briefly review Langland-Hassan's theory on Guiding Chosen imaginings, and highlight how the processes described in it can be mapped to two other theories strongly focused on the dynamics of imagination acts. In Section 3 we propose a refined framework that allows to account for a more detailed distinction regarding the dynamics of creating and developing imaginary worlds in formal settings, and we provide an example of how the framework can be used. Then, in Section 4 we introduce the Rhombus of Imagination and show how it can capture the procedural structure of different kinds of imagination acts. Finally, we summarize our main conclusions and point to some possible lines of future work in Section 5.

\section{The Dynamics of Imagination}

In this section, we start by reviewing the mechanisms identified in Peter Langland-Hassan's theory for Guiding Chosen imaginings. The quasi-formal treatment of Langland-Hassan's work towards the processes of imagination makes it a good starting point for our interest in drawing bridges between the dynamics of imagination and formal systems. Further on, we briefly point out how other previous works that focused on the dynamics of imagination identify similar mechanisms, and we take them as a starting point to distill a theory-independent, shared structure of dynamics processes.

\subsection{Langland-Hassan's Theory}

In Langland-Hassan (2016), Peter Langland-Hassan studies how it is possible that voluntary imagination acts initiated by our own intentions can be used to improve our epistemic state. The author distinguishes between four kinds of imaginings and focuses on Guiding Chosen (GC) imaginings, which are those imaginings voluntarily initiated by the agent, and which can provide useful 
information regarding how a certain imaginary situation would be, if it was real.

In order to understand GC imaginings, there are three features of their architecture that need to be accounted for: the initial involvement of topdown intentions that initiate an imagining, the use of lateral constraints in the development of such imagining, and the cyclical involvement of top-down intentions that are used to add new premises during the imagining.

When setting the initial content of an imagining, we use top-down intentions (that is: voluntary, conscious actions of the agent regarding what to imagine) that are indeed chosen. Langland-Hassan, then, focuses on how imaginative episodes "advance" from one state to another. As he claims, if only top-down intentions were used in the advancement of those episodes, then learning something new via imaginings would be pretty difficult, as their advancement would already be determined by our own will.

In order to overcome this problem, Langland-Hassan calls for a set of lateral constraints which, after the conditions of the initial imagining have been set, encode the set of norms, logic, or algorithms governing how the imaginative episode would unfold. Langland-Hassan identifies a sequence of mental states $i_{1}, \ldots, i_{n}$, which are altogether called an "imaginative episode", and in which each $i_{x}$ is called an "imaginative state". Now, the author sees each of the imaginative states $i_{x}$ in an imaginative episode as a result of an inference step, given what is the case in the previous state $i_{x-1}$, and following certain rules determining how $i_{x-1}$ leads to $i_{x}$. In particular, his account describes the unfolding of an imaginative scenario as a sort of step-by-step process guided by implications; for example, given an imaginative state $i_{x}$ with content $p$ and a set of lateral constraints containing a norm of the form $p \rightarrow q$, the next imaginative state $i_{x+1}$ would have content $q$.

Langland-Hassan refers to the "deviance" objection when taking into account how, even in cases of GC imagining, the way an imaginative episode advances usually deviates from anything we would likely expect to happen, given the initial conditions. While comparing his approach to Nichols and Stich's Script Elaborator (which we introduce in Section 2.2), the author notes how, if those deviant additions are understood as new premises being voluntarily added by the agent, then the role of the Script Elaborator could be reduced to a cyclical involvement of a new top-down intention that begins the whole process anew. In other words, Langland-Hassan argues that there is no need for a distinct mechanism devoted to this task. The new premises added in this case, then, would be determined by a desire of the agent to intervene in the imagining in a certain way.

The whole process of GC imaginings is represented in Fig. 1. The dotted circle at the left hand of the figure represents that there is no imaginary world yet; the dashed lines represent how certain processes of imagination either create a new imaginary world, or update an already existing one with new information. Note how this schema accounts for the fact that, whenever the scenario deviates in a "non-scripted" way through a new desire-driven addition, it is identified as being a new top-down intention. In particular, we 


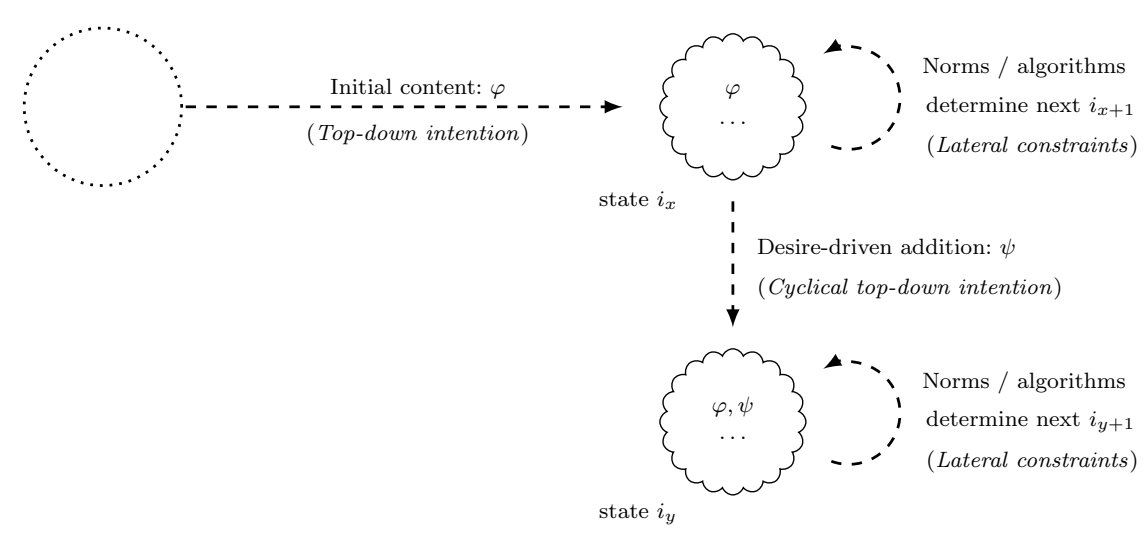

Fig. 1: An act of imagination according to Langland-Hassan's theory.

represent this by the fact that the state at the upper-right corner of the figure is identified as $i_{x}$ and, through applying the lateral constraints, it unfolds a next $i_{x+1}$ (which, in the schema, points back to the same state to capture the fact that those additions would "naturally" unfold in that state); however, and in order to stress out that a new top-down intention with a new premise will alter the imagining in atypical ways, the state at the lower-right corner of the figure is identified by $i_{y}$, instead of $i_{x+n}$. Once the new imagining has been set, though, lateral constraints compute the next $i_{y+1}$ by following the norms, rules and algorithms that describe how the imagining would typically unfold.

\subsection{Nichols and Stich's Cognitive Theory of Pretense}

Shaun Nichols and Stephen Stich present in Nichols and Stich (2000) a theory of pretense ${ }^{2}$ that aims to identify which mechanisms, within our minds, are involved in pretense episodes. The authors build their proposal upon an already existing architecture of the mind, which includes different "boxes" that store representation tokens accounting for beliefs and desires, as well as a set of inference mechanisms. Within this already existing architecture, Nichols and Stich introduce three further components needed to explain how our imagination works: the Possible World Box, a set of Inference Mechanisms and the Script Elaborator.

The Possible World Box (PWB onwards) is structurally and functionally very similar to the boxes containing belief and desire tokens. However, its main difference with respect to those is that the PWB's job is not to represent the world as it is, nor as the agent wishes it to be, but rather to represent how the world would be like, given a certain initial premise.

\footnotetext{
2 It is worth noting how, on page 127, the authors explain that, without taking action into account, their theory of pretense can be understood as a theory of imagination, which is in fact where our interest lies in this work.
} 
When the initial premise is added into the PWB, the cognitive system starts filling up the PWB with a detailed description of how the world would be like, if the pretense premise was true. This is handled by what they call the Inference Mechanisms, which are responsible for determining, given the initial premise and the agent's beliefs, what else would be the case in the imagined scenario. Those alternative developments of the initial imagining are then dumped into the PWB, and a sub-mechanism called the UpDater checks all the representations in the PWB, while deleting those that are not compatible with the new premise that has just been added.

Aside from the way things could typically unfold, there are still many ways in which a pretense episode could develop and which are neither specified by the initial premise, nor by the pretender's beliefs. Although the authors do not provide a detailed account of its structure and the way it works, they claim that there exists some mechanism (their emphasis) sub-serving this process, which they call the Script Elaborator. As we mentioned in Section 2.1, LanglandHassan's theory interprets this process as a new initiation of an imaginary episode.

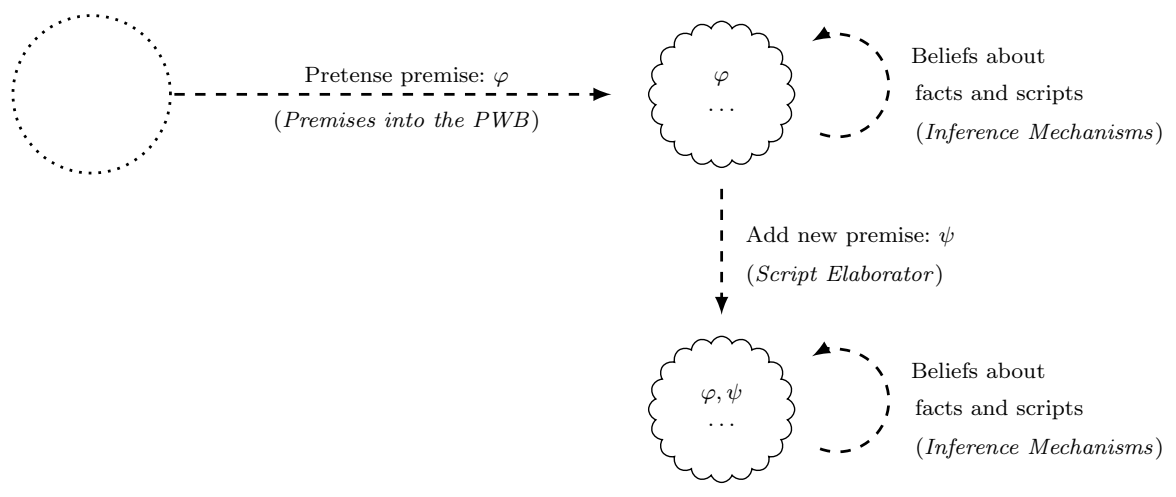

Fig. 2: An act of imagination according to Nichols and Stich's theory.

Fig. 2 represents how Nichols and Stich's theory accounts for the way imaginary scenarios are created and elaborated. A new scenario is initiated by putting a pretense premise $\varphi$ into the PWB, and then the scenario is filled up with details taken from the agent's beliefs, expressed as reality-oriented facts and scripts. Then, the Script Elaborator may also come up with a new pretense premise $\psi$, which is added into the scenario.

\subsection{Williamson's Modes of Imagination}

Further similarities regarding the dynamics of imagination can be found in Williamson (2016), where Timothy Williamson argues against the apparent 
opposition that exists between knowledge and imagination ${ }^{3}$, and that often appears in the literature. According to this distinction, knowledge is related to facts, whereas imagination is related to fiction. However, imagination can be, when used properly, a powerful tool to acquire new knowledge. In order to be used in such a way, imagination must be constrained by reality-oriented rules and facts that make imaginary scenarios unfold the same way they would do, if they were real. The author proposes a distinction between two different modes in which imagination can work: voluntary and involuntary.

In voluntary mode, certain initial conditions that describe an imaginary scenario are set. For instance, if someone wants to assess whether she would be able to jump through a mountain stream, the imaginary scenario should be initiated by including that mountain stream, plus any other detail that might be relevant for the assessment of the person's chance to jump over it.

Once these initial conditions have been set, imagination starts running in involuntary mode and unfolds the consequences that would likely follow from the initial scenario, if it was real. The epistemic value of these imaginary exercises lies in the "if it was real" part: of course, one can choose to imagine (almost) any outcome she wants, but it would be of little epistemic value if such outcome did not follow what would likely happen in the imagined scenario.

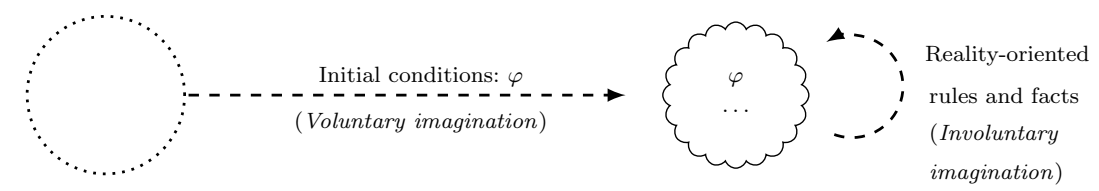

Fig. 3: An act of imagination according to Williamson's theory.

Fig. 3 represents how acts of imagination work, according to Williamson. Through an act of voluntary imagination with initial conditions $\varphi$, an imaginary world is created. Then, it is developed by the involuntary imagination, which follows reality-oriented rules and facts. Note how, unlike in Fig. 1 and Fig. 2, the schema for Williamson's theory does not account for any kind of atypical development of the imaginary world; this is in accordance with the fact that, in his work, the author is only concerned with reality-oriented development.

\subsection{Distilling the Theories}

Although the theories just reviewed have some differences, the analysis they do of the mechanisms involved in creating and developing imaginary worlds

\footnotetext{
3 It is worth noting that, as argued in Spaulding (2016), Williamson uses the term "imagination" in a broad sense, which includes supposition, the evaluation of conjunctive conditionals, and so on.
} 
have a lot in common. The reviewed theories all focus on voluntary acts of imagination. Every act of imagination characterized as such starts with a voluntary action of the agent that creates, or initiates, a new representation of an alternative state of affairs. This action requires, as a parameter, a set of initial premises, or conditions, that characterize the imaginary scenario that is to be created. This process corresponds to Langland-Hassan's initial top-down intentions, to Nichols and Stich's action of putting a premise into the PWB, and to Williamson's voluntary imagination.

Once the imaginary scenario has been created, it can be elaborated by providing more details of what else could be the case in there, with respect to the initial premises that have just been used to create it. The sources of such development are, again according to all three theories, encoded into the agent's beliefs and knowledge about the real world in the form of rules or scripts. We refer to this process as the reality-oriented development, and we associate it with Langland-Hassan's lateral constraints, Nichols and Stich's Inference Mechanisms, and Williamson's involuntary imagination.

More often than not, imaginative episodes develop in highly unexpected ways. Why does this happen, and where do these plot twists come from? The agent wishes something unexpected to happen, and thus decides to stop focusing on what would typically be the case in that scenario, and starts feeding new premises into the imaginary world. Only two of the three previous theories account for this action: as Williamson is only interested in reality-oriented imagination, his work does not account for this phenomenon. Nevertheless, both Langland-Hassan's theory, through the cyclical involvement of top-down intentions, as well as Nichols and Stich's theory, through the Script Elaborator, do take this into account.

Table 1 summarizes the relation between the identified mechanisms and each of the previously reviewed theories.

\begin{tabular}{|c|c|c|c|}
\hline & Langland-Hassan & Nichols / Stich & Williamson \\
\hline Voluntary initiation & Top-down intention & Premises into PWB & Volunt. imag. \\
Real. -orient. dev. & Lateral constraints & Inference Mechanisms & Involunt. imag. \\
Atypical dev. & Cyclical top-down int. & Script Elaborator & - \\
\hline
\end{tabular}

Table 1: A common underlying structure for the theories of imagination.

Although we can see how the reviewed theories agree on an underlying structure for the mechanisms of imagination, in the next section we argue how, in order to provide a suitable basis for representing the dynamics of imagination using a formal system, those mechanisms should be further refined. 


\subsection{Existing Logics of Imagination}

When considering formal systems, few authors have ventured into the uncharted seas of logic and imagination. In Lewis (1973), the author defines a logic to account for counterfactual reasoning by using a system of spheres and a modal operator that moves the evaluation point to counterfactual worlds. Later, Niiniluoto (1985) formalizes imagination as a propositional attitude and discusses some of its properties. In Costa-Leite (2011), the author goes one step beyond and formalizes the distinction between "imagination", "conception" and "possibility" through following the intuitions of Descartes and Hume. Wansing brings beliefs into the picture in Wansing (2017) and uses neighborhood semantics and STIT mechanics to account for agentive imagination. In Priest (2016), the author focuses on imagining impossibilities and defines a system that uses impossible worlds to model that. Through his works Berto (2014), Berto (2017) and Berto (2018), Berto formalizes conceivability in both a paraconsistent and a classical setting, and introduces the mechanics of "aboutness", which determine what is relevant for the agent to import conceiving an alternative world. In relation to his work on aboutness, Giordani (2019) defines an axiomatic system and proves its completeness.

Even though these works highlight very interesting features of imagination, they all represent imagination in static, pre-determined scenarios, like snapshots of a specific moment. Although Wansing's approach in Wansing (2017) goes one step beyond and takes into account the agentive character of imagination, it still works in predefined, tree-like structures: the agent can be seen as "choosing" what to imagine, indeed, but these choices are already contained in the initial model of the situation. Our approach aims to provide a framework to capture something that has been overlooked in these previous logical systems: imagination is, in essence, dynamic. Although we do not aim to define a fully-fledged logical system, we argue that the algorithmic analysis we provide for the dynamics of imaginary worlds can be used as a starting point to define a logic that allows to expand its formal models dynamically.

\section{The Common Frame for Imagination Acts}

Although the mechanisms identified in the previous theories may seem precise enough at first glance, we claim that they are not as specific as we need in order to fully account for the dynamics involved in imaginary worlds. This is particularly true if we aim to capture those dynamics using a formal language or a computer-based simulation. In particular, we claim that the reality-oriented development embeds two different mechanisms that should be distinguished, when aiming for a precise distinction of the processes involved in imagination acts. An imaginary scenario can mirror the real world in many different ways. Consider, for instance, an agent who decides to imagine a chessboard. Among other reasons, she could be imagining a chessboard because:

1. She wants to paint one upon a canvas. 
2. She wants to decide which her next move in a game of chess, given a certain setting of the pieces on the board.

According to each of these possibilities, the agent could develop her initial imagining by:

1. Filling up the details about how the chessboard is; for instance, whether it is made of wood, what color it is, and so on.

2. Considering the possible moves she could make and evaluating their consequences.

Now, in the first case, should the agent be concerned with what could happen in that scenario, meaning how the pieces could be moved across the board during a game? If she is just imagining the chessboard because she wants to paint one, then it would be of no use to go beyond the static details of the scenario. Regarding the second case, should the agent be concerned with how the chessboard is, like whether it is made of wood, or what material are the pieces made of? If she just wants to assess which is her next best move, it would be useless to focus on the static details of the chessboard, or its pieces.

When giving a closer look to how the existing theories of imagination define reality-oriented development, we notice how this mechanism actually embeds different ways of developing an imagining. As we see it, one could elaborate the static details of the imagining (which would correspond to case 1 in the previous chessboard example), without considering any kind of dynamic action that could typically take place in it (which would correspond to case 2), and the other way around. Although they both use reality-oriented inputs as their parameters, the way they develop an imagining is different enough to draw a distinction between them, specially if we aim to encode these mechanisms by using a formal, or algorithmic framework. On a formal level, the main distinction between both cases would be that, in the second case of our example, the system would need to take into account an action or event taking place in there, while it would not need to do so in the first case. That is, the dynamic elaboration of an imaginary scenario involves a certain change that takes place in the imagining as a result of something happening there, whereas the static elaboration just focuses on providing more details about a state of affairs where nothing "happens".

In the following subsections, we identify a refinement of the processes of imagination and we group them under what we call the Common Frame for Imagination Acts, which is a framework specially aimed at identifying and distinguishing the different mechanisms involved in creating and developing a representation of an imaginary world. Furthermore, we claim that this refined framework is better suited as the basis for studying the dynamics of imagination acts at a formal level. Once our proposal has been introduced, we show where the previously-reviewed theories match with it, and where they are actually collapsing distinct processes into a single mechanism. 


\subsection{The Initialization}

We call the first process of our frame the Initialization, and we associate to it the question "what characterizes the initial scenario?". It represent the agent performing a voluntary mental action ${ }^{4}$ that creates a new imaginary world, represented as a certain state of affairs fulfilling some initial premise. This premise may be more or less specific; for instance, it can either be something like "I am the singer of a jazz band", or "I am the singer of a jazz band formed by a piano, a double-bass and drums, where we are all wearing classy tuxedos". Either way, and be it more or less initially detailed, a new imaginary world will be created.

Following the previously reviewed theories of imagination, this mechanism aims to capture a kind of ceteris paribus imagination. In a nutshell, this means that, aside from what the agent is voluntarily choosing as the content of the new imagining, everything else is meant to be the same way as it is in the real world - that is, as long as it is consistent with the content of what is being imagined. In this sense, the Initialization process aims to create a "copy" of the real world in which only the content of the initial premise, as well as everything else needed to make that content consistent, is different from that real world ${ }^{5}$. Although authors such as David Lewis, in Lewis (1973), discuss in detail some issues that accommodating new content in a truly ceteris paribus setting could have, those concerns fall outside the scope of our current work and we leave them as future considerations.

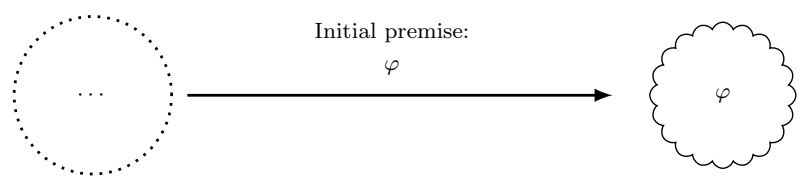

Fig. 4: The Initialization creates a new imaginary world, given an initial premise.

Fig. 4 depicts the Initialization process, in which a new imaginary world is created by using an initial premise $\varphi$. In order to provide a formal schema for this process, we need to take into account the initial premise $\varphi$ and the state of affairs representing the real world $w_{R}$, as believed by the agent that carries out the process. Then, the Initialization algorithm, which we call $A l g^{I}$, must create a new, empty representation of a possible world, and clamp the initial

\footnotetext{
4 As the author points out in Balcerak-Jackson (2016), succeeding in such voluntary action may vary depending on whether we are supposing, imagining or conceiving.

5 Note that, if possible worlds are used as formal models, imaginary worlds will then be required to be formally consistent. Nevertheless, different authors, such as Priest (2016), Berto (2017), or even Nichols and Stich in Nichols and Stich (2003) argue that one can also imagine impossible scenarios. If we wanted to allow for paraconsistent imaginings, our approach could be adapted to accommodate the use of impossible worlds in the way they are used, for instance, in Berto (2017).
} 
premise $\varphi$ in it - which we identify as the set of atomic formulas describing its content ${ }^{6}$ of the formula $\varphi$. We can identify the mathematical structures needed for this process as follows (for At being the set of atomic formulas of the given formal language):

- Input:

Real world: $\quad w_{R}=\left\{p_{i} \mid p_{i} \in A t\right.$ and $p_{i}$ is the case in the real world $\}$ Initial premise: $\varphi$ (formula of the formal language)

- Algorithm:

$\operatorname{Alg}\left(w_{R}, \varphi\right) \rightarrow w_{\varphi}$, where $w_{\varphi}$ is the imaginary world created by clamping the set of atoms $p_{j}$ accounted for in $\varphi$.

- Output:

$$
w_{\varphi}=\bigcup\left(p_{j} \mid p_{j} \text { is accounted for in } \varphi\right)
$$

Intuitively, the Initialization process requires an initial premise $\varphi$ that the agent wants to imagine, as well as a real world $w_{R}$ which will be used as the world of reference from which the agent started the imagining. The algorithm $A l g^{I}$, then, takes the two input parameters and computes, as the output, a new imaginary world $w_{\varphi}$. This new imaginary world is characterized by accounting for the state of affairs represented by the initial premise $\varphi$. Although the world of reference is not used in this step, world $w_{R}$ represents the state of affairs the agent believes to be the case, and, as such, contains those believed factual rules and scripts that will be used in further processes to elaborate on the details of the imagining.

\subsection{The Description}

This second process, which we call the Description, and which answers the question "what else is the case in the imaginary scenario?", corresponds to the "static" part of the development of an imaginary scenario. This process involves filling up the details of how the initial imagining could be like, regarding what other facts could also be the case in there, but without moving it into a new, different scenario that follows from any event, or action. We call the kind of rules governing the static description of an imaginary scenario the factual rules.

For example, an imaginary scenario initiated by the premise "we are having a tea-party" may be enriched with details about the shape of the tea-pot, the presence or absence of cookies on the table, the room we are in, the way people are dressed, etc. Note how these new details are about the current scenario

\footnotetext{
6 In the formal definition, we use the expression " $p_{j}$ is accounted for in $\varphi$ " to express the fact that the truth conditions for the formula $\varphi$ determine the truth-value of the atomic formula $p_{j}$ by requiring it to be either true or false in the state of affairs described by $\varphi$.
} 
we are describing: we are not yet putting our imagining in motion, but just forming a more detailed static version of the initial scenario.

We could draw an analogy with the language of propositional logic ${ }^{7}$, in which factual rules would correspond to formulas of the sort $t \rightarrow c \vee k$ : "if there is a tea-party, then there are also cookies or cake". Note how, when considering the previous formula in a possible-worlds semantics, atomic formulas $c$ and $k$ refer to the same state that satisfies $t$ : we are just adding details to it.

At this point is where our proposal starts departing from the previously reviewed theories, and starts being also more detailed than them. Although, when considering reality-oriented development, Nichols and Stich do mention that both facts and scripts are involved in this process, they leave them both to be handled by the Inference Mechanisms ${ }^{8}$, without providing any specific details on how similar, or distinct the development using facts or scripts should be. Langland-Hassan's theory behaves similarly to Nichols and Stich's, and he attributes this static description process to the lateral constraints which, again, are the same ones that govern how the scenario would typically unfold, regarding the actions or events that could take place in there. The same applies to Williamson's theory: involuntary imagination handles both the description of the imagined scenario, and unfolding the way it would typically evolve. The framework we are just defining, then, should be seen as a more precise account of the differences between distinct processes involved in the so-called reality-oriented development, and which are not made explicit enough in the previously reviewed theories.

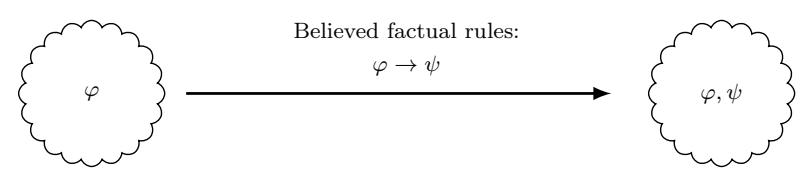

Fig. 5: The Description process elaborates on the static details of the scenario.

Fig. 5 represents how the Description process works ${ }^{9}$. Note how this process also uses a world of reference as a starting point, but, conversely to the case of the Initialization process, this one needs the world of reference to be imaginary. We refer to the world of reference as $w_{\varphi}$. For the sake of clarity, this would could be interpreted as being the imaginary world created during the Initialization

\footnotetext{
7 For a comprehensive introduction to propositional logic, see Hodges (2001), for instance.

8 Particularly, on page 118 of Nichols and Stich (2000), they identify both processes as the inferential elaboration, without distinguishing between their static or dynamic character.

9 It is important to note that Figure 5 is not meant to be a Kripke model, but rather just a schematic representation of the process. As such, and in order to stress out the fact that this process elaborates on the details of the same imaginary world, we represent believed factual rules using standard propositional logic and without any kind of modal operator. Figure 5, then, aims to represent how the world on the left changes into the world on the right, rather than representing that both worlds are different, in terms of possible-worlds semantics. For more details on modal logic, see Blackburn et al. (2010).
} 
process: after all, one cannot elaborate on the details of an imagining, unless such imagining has been already created.

In this process, the agent starts to elaborate on how the details of that initial imaginary world would be, considering what would follow from the initial premise used in the previous process. In this case, however, instead of using formula $\varphi$ as the premise to create a new imaginary world, the agent would browse her believed factual rules, to which we formally refer as a set Fact, in order to see what else would be the case in the imaginary world, given $\varphi$. In this process, we refer to $\varphi$ as the focus the agent uses to unfold what else would be the case in the imagining. In particular, the agent's factual rules correspond to a set of rules that represent expressions of the $\operatorname{kind}^{10}$ "if $\varphi$ were the case, then $\psi$ would also be the case".

- Input:

Imaginary world: $w_{\varphi}$ (an already existing imaginary world)

Focus: $\quad \varphi$ (the initial premise used to create $\left.w_{\varphi}\right)$

Believed fact. rule: $\zeta \in$ Fact, where $\zeta$ has the form $(\varphi \rightarrow \psi)$

- Algorithm:

$\operatorname{Alg}^{D}\left(w_{\varphi}, \varphi, \zeta\right) \rightarrow w_{\psi}$, where $w_{\psi}$ is the imaginary world expanded by adding those additional facts $p_{i}$ that can be inferred from having $\varphi$, and as specified by the factual rule $\zeta$, as well as removing those facts $p_{j}$ that would contradict these new facts $p_{i}$.

- Output:

$$
\begin{array}{r}
w_{\psi}=\bigcup\left(p_{i} \mid p_{i} \text { is accounted for in } \psi\right) \cup \\
\bigcup\left(p_{j} \mid p_{j} \in w_{\varphi} \text { and }\left(p_{j} \wedge \psi\right) \not \equiv \perp\right)
\end{array}
$$

\footnotetext{
10 Note that, although this kind of expressions can be mapped into Lewis' account of counterfactuals in Lewis (1973), the way they are used in our approach is different. Whereas Lewis' approach of such expression would "move" the point of evaluation to a different world where both antecedent and consequent are the case, our approach "breaks down" the evaluation in two different steps. First, it checks whether the antecedent $i s$ already the case in the current world and, if so, then creates a new world, which represents a refinement of the current one, and in which the consequent also holds. The notion of "refinement" can be, in fact, a bit controversial when thinking about possible worlds; after all, possible worlds are maximally consistent sets of formulas in which each and every atomic formula is already assigned a truth-value. We use the term "refinement" is a more informal or intuitive way. Even if the truth-value of all atomic formulas needs to be set in our imaginary worlds, their truth-value may be the result of importing it from the world of reference, instead of determining what is should be by looking at how the imagining develops. In this sense, and even if the technical apparatus behind a classical approach requires all atomic formulas to have a truth-value, by "refining" the world we mean that, by pulling onto certain factual beliefs, the agent "realizes" that the truth-value of certain atomic formulas should change in the imagining, with respect to what they were before. In this sense, "refining" a world could be understood as "updating" the truth-values of certain atomic formulas step-bystep, given the factual rules believed by the agent. Those "refinement" steps could be seen as specific executions of some of the broader sets of inferential mechanisms identified by previous authors.
} 
On an intuitive level, the Description process takes an already existing imaginary world $w_{\varphi}$, created through imagining an initial premise $\varphi$, and elaborates on what else would be the case in the imagining by considering $\varphi$ and a factual rule $\zeta$ of the form $\varphi \rightarrow \psi$. In this case, algorithm $A l g^{D}$ adds to the imagining those atomic formulas $p_{i}$ that account for $\psi$, while removing those atomic formulas $p_{j}$ that already exist in the imagining, and which would contradict the new ones. The new imaginary world $w_{\psi}$, then, is defined following two different steps:

1. The atomic formulas $p_{i}$ belonging to the new content $\psi$ have priority over what was already the case in the world used as the input, and therefore are clamped firstly.

2. Then, the atomic formulas $p_{j}$ that were the case in the world $w_{\varphi}$ used as the input are kept into the new world, as long as they do not contradict with any of the atoms $p_{i}$ clamped in there.

This same procedure when elaborating the details of an imaginary world will be followed in the following processes in order to prioritize the new content being imagined, while ensuring that the resulting imaginary world, once the rest of the atomic formulas have been imported, is consistent with those.

\subsection{The Default Evolution}

We call the third process the Default Evolution, and we associate it to the question "what would typically happen in the imaginary scenario?". The main point of this process is to determine, still by using reality-oriented inputs based on the agent's beliefs, how the scenario could typically evolve, or "move forward", regarding what events or actions could take place in there. We call the kind of inputs describing the possible ways a scenario could move forward the scripts ${ }^{11}$.

For instance, in the previous example initiated with the premise "we are having a tea-party", our believed scripts about tea-parties could tell us something about how the scenario may typically "move forward", how people would typically behave, or what actions could take place in there. A few examples could be, for instance, "if I am in a tea-party, I could pour tea into my cup", or

\footnotetext{
11 In Funkhouser and Spaulding (2009), the authors use the term "script" to refer to something that can be imitated or enacted, and that can guide action without the need of being backed up by beliefs or knowledge. Our use of the term differs from theirs in the sense that, in our account, scripts are not meant to guide an actual action, but rather to set, according to the current state of the imaginary scenario, which actions or events could take place in there. In particular, our scripts encode something like "in a situation in which such and such was the case, executing action $\alpha$ could lead to this set of outcomes". Scripts, then, are neither guides to enact an action, nor a way to assign a tendency to certain actions to happen in an imaginary scenario, but rather a way of determining what an action could bring about, given the conditions of such scenario. Therefore, although both our and their use of the term "script" has some similarities, as in both cases it concerns actions that could happen in an imaginary scenario, in our setting scripts are just meant to open new modal paths opened up by certain actions or events.
} 
"if there is a tea-party, everyone is going to sip tea slowly, in a well-educated manner". Note how, with respect to the factual rules used by the Description process, these scripts do not provide information regarding what else could be the case in there - meaning what other facts could be true in that state of affairs $^{12}$, or how the scenario could look like. Instead, they rather tell us what could happen, or what I could do in that scenario. They are not tokens that provide further details about the state of affairs of the current static scenario, but rather tokens that describe how some "accessible", "possible", or "future" scenarios based on the current one could be reached through the occurrence of a certain action or event. Conversely to the Description process, where the agent chooses to focus on certain static details (represented by a certain logical formula), the focus in the Default Evolution process is instead the action that the agent imagines to happen in the imaginary world, and which would bring about a series of changes to the state of affairs.

When considering how this would be expressed using a formal setting, we find that actions are often represented as being transitions between states, rather than states (or part of states) themselves. If we compare, for instance, Propositional Modal Logic (we refer again to Blackburn et al. (2010)) with Propositional Dynamic Logic, or PDL (see Harel (1984)), we can see that one of the main differences between both is that PDL identifies actions (called "programs" in PDL) as the triggers for changes between state of affairs. Moreover, in PDL these actions are bind to the accessibility relations between different states, rather than to the states themselves. In this sense, actions are represented in such a way that they cause a change between two different "static" states of affairs. Following this, we interpret the scripts responsible of the Default Evolution process not by having actions as part of their "final state", such as $t \rightarrow \diamond p$ (where $p$ corresponds to the action "pour tea into my cup"), but rather by being "state changers" that bring about a different situation, such as $t \rightarrow\langle p\rangle f$, where $t$ correspond to "there is a tea party", $p$ corresponds to the action "pour tea into my cup", and $f$ corresponds to the fact "my cup is full". Note that, interpreted in such a way, actions or events are not the outcomes of our scripts, but rather the way and the cause that affects the current state of affairs in some way. The main difference between the scripts

\footnotetext{
12 It could be argued that, in fact, scripts do provide some information regarding what actions are most likely to occur in a certain scenario. Nevertheless, the structures we use to define our approach do not allow to capture, at least not explicitly, this notion of "typicality", or "likeliness" for an action to happen in a given scenario. Instead, our approach assumes that the agent already has scripts about every possible action available; what makes an action "executable" in a certain scenario, then, is determined by the conditions that the script should fulfill in order to bring about its consequences. More intuitively, the action "pour tea into a cup" would only have an actual effect in those scenarios in which, for instance, there is a cup and a tea pot, but it will not affect, say, a scenario where there are only apples and oranges on a table. Even though our approach, as it is, cannot yet account for updating the likeliness of certain actions to be imagined in particular scenarios, it could be extended by including a kind of "sorted preference list" over scripts for the imagining being entertained. The list would have to be ordered in such a way that, the closer the current imagining is to the preconditions of a particular script, the more relevant this script becomes in the list, and thus the more likely it is to be selected by the agent.
} 
used in this process, and the factual rules used in the Description process, is that these scripts do not tell us anything new about the same scenario in which $t$ is the case, whereas the factual rules of the Description process do. The scripts, instead, require that a certain event or action takes places, and thus they require that the current, static "snapshot" of the state of affairs moves forward.

Again, and as a consequence of not distinguishing between the static and the dynamic elaboration of an imaginary scenario, this process is also embedded into Langland-Hassan's lateral constraints, into Nichols and Stich's Inference Mechanisms, and into Williamson's involuntary imagination.

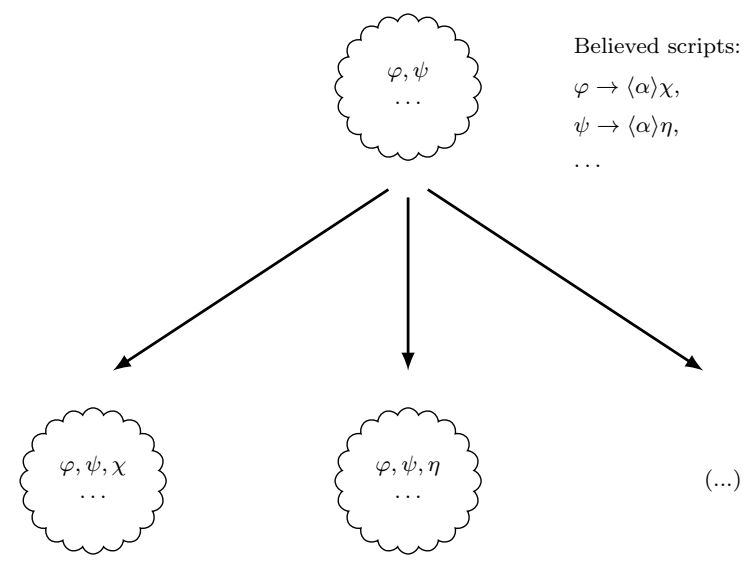

Fig. 6: The Default Evolution process opens possible outcomes of the scenario.

Fig. 6 represents the Default Evolution process. Note how, unlike the Description process, in this case the scripts are not used to elaborate on the details of the current imaginary scenario in a static way, but rather to evaluate the possible ways in which such scenario could "evolve", or move forward. Obviously, a specific scenario could usually move forward in many different ways; it is up to the agent, then, to decide which course of events she wants to choose in her imagining. Similarly to the case of the Description process, though, the agent elaborates on the details of an already existing imaginary world. The difference in this case, however, is that the Default Evolution captures a dynamic change in the imaginary scenario - that is, a change caused by a certain event happening, or an action being carried out by the agent involved in the imagining.

- Input:

Imaginary world: $w_{\psi}$ (an already existing imaginary world)

Focus (Action): $\quad \alpha$ (a formula of the language accounting for an action)

Believed scripts: $\left\{\theta_{k}\right\}^{\alpha} \subseteq$ Script, where each $\theta_{i}$ has the form $(\gamma \rightarrow\langle\alpha\rangle \delta)$, and where $\alpha$ is the action the agent imagines to happen. 
- Algorithm:

$\operatorname{Alg}{ }^{E}\left(w_{\psi}, \alpha,\left\{\theta_{k}\right\}^{\alpha}\right) \rightarrow\left\{w_{\chi_{i}}\right\}$, where $\left\{w_{\chi_{i}}\right\}$ is the set of imaginary worlds and each $w_{\chi_{i}}$ is an imaginary world expanded by adding the atomic facts $p_{k_{j}}$, according to each script $\theta_{k}$ of the form $\gamma_{k} \rightarrow\langle\alpha\rangle \delta_{k}$, as a result of the agent imagining that action $\alpha$ happens, and provided that the antecedent $\gamma_{k}$ is the case in world $w_{\psi}$.

- Output:

$$
\begin{aligned}
&\left\{w_{\chi_{i}}\right\}, \text { where each } w_{\chi_{i}}= \bigcup\left(p_{j} \mid p_{j} \text { is accounted for in } \delta_{i}\right) \cup \\
& \bigcup\left(p_{k} \mid p_{k} \in w_{\psi} \text { and }\left(p_{k} \wedge \delta_{i}\right) \not \equiv \perp\right)
\end{aligned}
$$

Regarding the intuitions behind this process, the agent takes an imaginary world $w_{\psi}$ as the starting point (where other processes of imagination, such as the Description process, could have already happened) and then browses over her believed scripts. These scripts be expressed using formulas of the form $\gamma \rightarrow\langle\alpha\rangle \delta$, which capture that "if $\gamma$ were the case, and event $\alpha$ were to happen, then $\delta$ could also be the case". In this case, then, the agent does not take as focus a formula of the world of reference, but rather she imagines that an action $\alpha$ happens in the imagining. As a result, the agent must browse over all her beliefs regarding action $\alpha$ and then, whenever the antecedent of such beliefs is met in the world of reference, elaborate on the consequences that such action would have on the imagining. $A l g^{E}$ does that and, as a result, might return as an output not just a single imaginary world, but possibly a set of different imaginary worlds, depending on the scripts believed by the agent. Similarly to what happened in the Description process, the atomic formulas imported from the world of reference would give priority to the new atomic formulas just added in order to ensure consistency.

\subsection{The Unscripted Additions}

We call the last process identified in our frame the Unscripted Addition, and we associate it to the question "how does the agent voluntarily change the scenario?". This process corresponds to those ways of developing an imaginary scenario that are not typical, and which would not follow from reality-oriented rules or scripts.

Note how the question with which we characterize this process strongly emphasizes the voluntary intention of the agent to go "off-rails" with additions that deviate from what one would expect to be the case, or happen in the imagining. As the agent chooses again a new premise to be put into the imaginary scenario, this process behaves like the Initialization process of our framework ${ }^{13}$. The agent adds a new premise into an already existing imaginary scenario, and then the cycle of filling up the details, imagining how the

\footnotetext{
13 In Fact, Langland-Hassan argues that these kind of additions, which Nichols and Stich attribute to their opaque Script Elaborator, are nothing more than new premises that the agent clamps to the imaginary scenario.
} 
scenario moves forward, and coming up with yet another off-script premise, begins again.

Unlike the Initialization process, which is responsible for creating a brandnew imaginary scenario, these additions are built upon an already existing imaginary scenario that is already specified up to a certain point. The new premises added by this process may be completely new, but they may still override something already set in a previous imagining. If the new premise can be held together with what is already the case in the imagining, so be it; otherwise, it should override whatever was already the case in there that conflicts with it. In other words: these new premises have priority over everything else.

This process can be mapped into Langland-Hassan's cyclical involvement of top-down intentions and into Nichols and Stich's Script Elaborator. As we have already argued, Williamson's theory does not consider this mechanism of imagination acts, as he is interested in the development of imaginary scenarios that follow reality-oriented rules and facts.

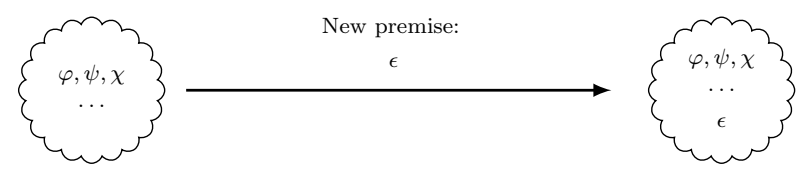

Fig. 7: The Unscripted Addition adds a new premise into the imaginary world.

Fig. 7 represents the Unscripted Addition process. Note how this process is, in accordance to Langland-Hassan's theory, pretty similar to the Initialization. These kind of additions are neither implied by any kind of factual rule, nor script; instead, they are voluntarily added by the agent. The reason for adding a new premise into the imaginary scenario follows a desire to elaborate the scenario in a certain way. In this case, the agent chooses an already existing imaginary world $w_{\chi}$ and a new premise $\epsilon$ to voluntarily clamp into it. The resulting world should account for this new clamped premise $\epsilon$ and should import every other atomic fact that is the case in the world of reference $w_{\chi}$, as long as it does not contradict with the new information being added.

- Input:

Imaginary world: $w_{\chi}$ (an already existing imaginary world)

New premise: $\quad \epsilon$ (formula of the formal language)

- Algorithm:

$\operatorname{Alg}^{A}\left(w_{\chi}, \epsilon\right) \rightarrow w_{\epsilon}$, where $w_{\epsilon}$ is the new imaginary world created by clamping the set of atoms $p_{i} \in \epsilon$, as well as importing those atoms $p_{j} \in w_{\chi}$ that do not contradict $\epsilon$. 
- Output:

$$
\begin{array}{r}
w_{\epsilon}=\bigcup\left(p_{i} \mid p_{i} \text { is accounted for in } \epsilon\right) \cup \\
\bigcup\left(p_{j} \mid p_{j} \in w_{\chi} \text { and }\left(p_{j} \wedge \epsilon\right) \not \equiv \perp\right)
\end{array}
$$

On an intuitive level, the Unscripted Additions mimics the behavior of the Initialization process, but with an important difference: the world of reference $w_{\chi}$, in this case, needs to be an imaginary world, instead of a real one. Then, the agent chooses a new premise $\epsilon$, which do not derive from any of her factual rules or scripts, and clamps it to elaborate the imagining into a new imaginary world $w_{\epsilon}$.

\subsection{Binding Everything Together}

Up to this point, we have identified and refined, with respect to the reviewed existing theories of imagination, what we claim are the four distinct processes involved in the creation and development of imaginary worlds that result from voluntary acts of imagination. Note, however, that most of these processes are not required to be performed in a sequential way; in fact, the only one that is indeed required to happen in a specific order is the first one, namely the Initialization. After all, without a voluntary mental action initiating an imagining, it would not be possible to develop such imagining any further. The other three processes, nonetheless, could be (and usually are) performed in a mixed way, without necessarily following any order.

The framework identified in the Common Frame for Imagination Acts can be summarized, in comparison to the previously reviewed theories, in Table 2. As we can see, the theories of imagination we have reviewed earlier fail to distinguish between the Description and the Default Evolution processes, and they collapse both within a reality-oriented development step. Even though these two processes are, indeed, related to the development of the imaginary world by following reality-oriented rules, we claim that, in order to capture and reproduce those dynamics in a formal way, these two processes should be clearly distinguished.

\begin{tabular}{|c|c|c|c|}
\hline [authors] & Langland-Hassan & Nichols / Stich & Williamson \\
\cline { 1 - 3 } Initialization & Top-down intention & Premises into PWB & Volunt. imag. \\
\cline { 1 - 2 } Description & \multirow{2}{*}{ Lateral constraints } & Inference Mechanisms & Involunt. imag. \\
\cline { 1 - 1 } Default Evo. & & & - \\
\hline Unscripted Add. & Cyclical top-down int. & Script Elaborator & - \\
\hline
\end{tabular}

Table 2: The Common Frame with respect to other theories of imagination.

The reasons behind why we think that separating the Description from the Default Evolution process is important are many-folded. 
On the one hand, and following previous works in formal logic such as Propositional Dynamic Logic (see Harel (1984)), we argue that actions, or events, should be distinguished from facts in a formal setting. While possible worlds aim to represent what is the case in a current state of affairs, actions are often seen not as "properties" of a certain state, but rather as statechangers, or transitions that affect a state of affairs in a certain way. This way of interpreting actions follows from the way Propositional Dynamic Logic, or PDL, represents "programs" as transitions between states. According to this interpretation, possible worlds represent "snapshots" of static scenarios in which the atomic formulas account for facts, whereas actions, or programs in the case of PDL, capture the cause that triggered a change in that static state.

On the other hand, we claim that it is important to distinguish both processes in order to implicitly bring the notion of time into the picture. In particular, an imaginary scenario that is being further developed in terms of what else could be the case in there does not need to "be put in motion". Like a painting, a depiction of a static state of affairs becomes more and more detailed without the need of time passing - at least not within the state of affairs itself. Conversely, whenever an action or event occurs, it occurs within a certain period of time, even if it is just a millisecond. Furthermore, those actions, and therefore the states of affairs they bring about, get implicitly set into an ordered sequence of static states and state-changers that potentially affect them in some way. Although time is not explicitly taken into account in our approach, distinguishing actions beforehand as transitions between states opens up the possibility of bringing time into the picture ${ }^{14}$.

This, in turn, potentially allows our models to be used for different purposes. As we argue in Section 4, certain uses of imagination may be mostly concerned with possible executions of actions - like planning in a game of chess. Similarly, other uses of imagination focus on a single, static scenario, with no, or little involvement of actions occurring over time. This can also be useful when imaginatively evolving a narrative backwards in time, or even to model some sort of abductive reasoning in which, given a certain state of affairs, the agent imaginatively follows possible courses of actions backwards in time.

We consider that our analysis of the dynamics of imagination acts can be taken as a suitable underlying framework for the modeling of how imaginary worlds are created and developed, when performing one of such acts of imagination. Moreover, we also claim that the Common Frame for Imagination Acts

\footnotetext{
14 It is only fair to note how, considering the way the Description algorithm works, it still creates a new state of affairs related via an accessibility relation. Nevertheless, and conversely to what happens with the Default Evolution, this transition is purely meant to capture a refinement of a static scenario, rather than an actual event occurring in the scenario being represented. In other words, these refinements can be interpreted as small "reasoning steps" similar to those that appear in Velazquez Quesada (2011). If a formal layer for time was to be added to our approach, only those transitions labeled with an action would represent an actual change in time, whereas those transitions resulting from an execution of the Description would still represent a scenario at the same time instant.
} 
can be used as a solid starting point when defining a formal system accounting for the way imaginary worlds are created, as we do in [authors]. Even though the previously reviewed theories already identified the main mechanisms involved in imagination acts, their analysis was not detailed enough to be translated into a formal or computational setting. Our proposal amends this caveat by being more specific, and splitting two processes embedded in the reality-oriented development mechanism.

When considering the formal details that each process would likely need to satisfy, we identified the inputs, algorithms and outputs that would account for each mechanism. Table 3 provides a general comparison between the sketched formal details of each process in order to provide an overview of their main similarities and differences on a mathematical level.

\begin{tabular}{|c|c|c|c|}
\hline Process & Input & Algorithm & Output \\
\hline Initialization & $w_{R}, \varphi$ & $\operatorname{Alg}^{I}\left(w_{R}, \varphi\right) \rightarrow w_{\varphi}$ & $w_{\varphi}$ \\
\hline Description & $w_{\varphi}, \varphi, \zeta \in$ Fact $\varphi$ & $\operatorname{Alg}^{D}\left(w_{\varphi}, \varphi, \zeta\right) \rightarrow w_{\psi}$ & $w_{\psi}$ \\
\hline Default Evo. & $w_{\psi}, \alpha,\left\{\theta_{k}\right\}^{\alpha} \subseteq$ Script & $\operatorname{Alg}^{E}\left(w_{\psi}, \alpha,\left\{\theta_{k}\right\}^{\alpha}\right) \rightarrow\left\{w_{\chi_{i}}\right\}$ & $\left\{w_{\chi_{i}}\right\}$ \\
\hline Unscripted Add. & $w_{\chi}, \epsilon$ & $\operatorname{Alg}^{A}\left(w_{\chi}, \epsilon\right) \rightarrow w_{\epsilon}$ & $w_{\epsilon}$ \\
\hline
\end{tabular}

Table 3: The Common Frame with respect to other theories of imagination.

\subsection{Remarks on the Description and the Default Evolution}

In the previous pages we have identified two distinct processes, namely the Description and the Default Evolution, as being responsible for elaborating an imaginary scenario. This elaboration is done by following certain factual rules and scripts detailing, respectively, what else could be the case in the imagining, or what could happen in there. Our aim is to provide a detailed identification of the processes involved in imaginary worlds, but we do not provide any further insights on the issue of how, exactly, does the agent get to choose one over the other, as it would fall outside the scope of this work. If, while imagining being in a tea-party, the agent can both infer that there could be a cake, but also that there could not be one, what is it that makes him elaborate the imagining in one way, instead of the other?

In Walton (1990), the author explores the so-called Principles of Generation, which aim to answer the question of why we develop our imaginings in certain ways, and by following certain rules. Similarly, in Van Leeuwen (2013) the author explores the rules that govern constructive imagining, such as the Belief Governance Thesis or the Genre Truth Governance Thesis, and which determine how, and why, we infer new details in them. In a similar way, but specifically focusing on pretense, Rucińska (2016) explores the same issue. 
As we have said, the aim of the present paper is to identify the processes used when creating imaginary worlds, and specifically to distill a structure than can be later translated into a formal setting. Due to this, we do not deal with what specifically guides the choosing of one rule over the other. Instead, we refer to the literature mentioned in the previous paragraph for more on this topic.

Furthermore, and even though we do not aim to provide a detailed definition of the algorithms in the present work, there are a few particularities and requirements that those algorithms would need to fulfill, and which we want to briefly point out.

Particularly, and being both reality-oriented and based on the agent's beliefs, the Description and the Default Evolution algorithms leave some open questions regarding what kind of algorithms they are, and how they could be encoded properly. Although the computational definition of "algorithm" is usually understood as being deterministic and deductive in nature, algorithms that aim to capture how background beliefs affect an imaginary scenario should probably be thought of as being more inductive in nature.

This, however, would depend on what particular facts, or actions, the rule or script is about. Taking scripts as an example, actions about which the agent has some knowledge, or a high degree of belief, would probably be encoded more deductively than those about which the agent has a softer form of belief. Examples of actions which consequences would be inferred deductively would involve, for instance, laws of nature, such as the laws of physics - that is, unless the imaginary scenario explicitly violates some of those rules. Conversely, actions about which the agent has no strong beliefs, or that somehow depend on skill, or chance, would not normally be expected to have a deterministic outcome - unless the conditions in which they are executed force it. Examples of such actions would include rolling some dice with their many possible outcomes, or trying to jump across a 2-meter wide mountain stream. The same would apply in the case of factual rules, of course. Whereas a rule such as "if there is a fire, is smoke" would be applied in a deductive fashion, a rule like "if there is a tea-party, there are cookies" is much less certain to hold in every tea-party scenario - regardless of how tasty it sounds.

Both the Description and the Default Evolution, therefore, must be able to account for factual rules and scripts that are more or less deductive, or inductive. This, however, could be handled not by the algorithm itself, but rather by the form of each specific rule and script. In particular, rules and scripts that aim to be deductive in nature would need to ensure that every possible world created as a result of executing the relevant algorithms fulfill the outcomes they are describing; the notion of "necessity", taken from alethic modal logic (see Blackburn et al. (2010)), captures this requirement. Similarly, rules and scripts aiming to capture a less strict, more inductive kind of rule would need to make use of the notion of "possibility", again taken from alethic modal logic, to represent that their outcomes is not so certain. It would even be possible, by using certain mechanics from probabilistic modal logic (see Shirazi and Amir (2007)), to represent how likely each outcome would be. 
When formally defining the algorithms, then, they should be made in such a way that they can distinguish, and act in accordance to, these two kinds of factual rules and scripts.

\subsection{An Example: Towards Modeling Thought Experiments}

In order to show how our framework could be applied to a formal representation of imagination acts, we briefly sketch how a commonly known thought experiment, used to prove that the universe was infinite (see Sorensen (1992)), could be modeled step by step by using our framework.

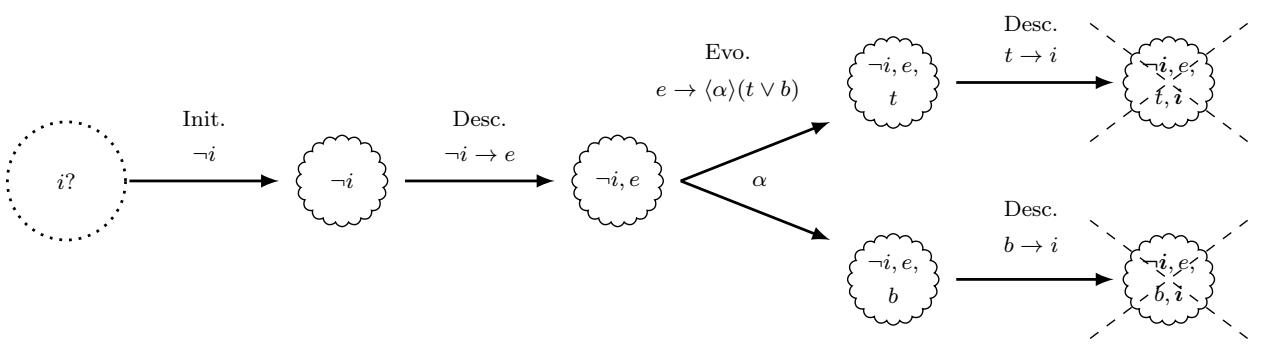

Fig. 8: Modeling thought experiments with the Common Frame.

Figure 8 shows how the thought experiment unfolds by applying the processes identified in our framework. The steps depicted in such figure, from left to right, correspond to the following ones:

1. Initially, the agent does not know whether the universe is infinite $(i)$ or not (note that we represent the initial world to be a real world by using a dotted line).

2. The agent initiates the thought experiment by imagining, or supposing, that the universe is not infinite $(\neg i)$ This is done through the Initialization process, and creates a new imaginary world with the initial premise $\neg i$.

3. Then, the agent uses one of her factual rules stating that "if the universe was not infinite, there would be an edge of the universe" $(\neg i \rightarrow e)$. This uses the Description process to elaborate on the static details of the new imaginary world focusing on $\neg i$, and concluding that $e$ would also be the case in such imagining.

4. Once the agent concludes that there is an edge of the universe in her imagining, she imagines what would happen if she threw a spear at the edge of the universe, represented by action $\alpha$. This, following her believed scripts about the consequences of $\alpha$, pulls the script $e \rightarrow\langle\alpha\rangle(t \vee b)$, which accounts for "if there were an edge of the universe, and I threw a spear at it, it would either go through $(t)$, or it would bounce back $(b)$ ". For the sake of clarity, we account for those two consequences separately by using two 
different imaginary worlds in Figure 8. This elaboration is done through the Default Evolution process.

5. Then, in order to see what else would be the case in the new imaginary worlds, the agent browses again over her factual rules to see that, firstly, "if the spear went through $(t)$, then the edge would be a limit between the universe and something beyond it (l)". This, again, uses the Description process. Similarly, in the other imaginary world, the agent uses the factual rule "if the spear bounced back $(b)$, then the edge would be a limit between the universe and something beyond it $(l)$ ", which also adds formula $l$ to the imagining.

6. Last, but not least, the agent uses the Description process again in both scenarios to conclude that, "if there was a limit between the universe and something beyond it $(l)$, then the universe would be infinite $(i)$ ", thus deriving $i$ in both imaginary worlds, and thus contradicting her initial assumption of $\neg i$ being the case (in Figure 8 we cross out the inconsistent worlds, while highlighting the contradictory formulas in bold font).

7. Although our framework does not account for the mechanics of "taking information back" to the real world, or learning, such example would need a way of taking that contradiction back to the real world in order to add the new information about the universe being, in fact, infinite (i), thus changing the information the agent had in the real world.

\section{The Rhombus of Imagination}

In this section, we introduce a visual tool aimed to provide a straight-forward way of representing up to which point different kinds of imagination acts rely on each of the processes identified in the Common Frame for Imagination Acts, and which we call the Rhombus of Imagination. By being able to compare, at a single glance, how similar and different some kinds of imagination acts are, with respect to their dynamics, we argue that it is possible to distill certain properties that characterize the procedural structure of those kinds of imagination acts.

The Rhombus of Imagination is formed by a main rhombus shape ${ }^{15}$, which we call the outer rhombus, and in which each vertex corresponds to one of the processes of the Common Frame for Imagination Acts. Each vertex is connected to the center of the outer rhombus by a line, which is used to measure how much a certain kind of imagination acts rely on using that particular process. Specifically, the farther away from the center of the rhombus, the more it relies on that process. We divide each line in three distinguished measures, each one corresponding to a certain degree of relevance:

1. The first measure, coinciding with the center of the outer rhombus, corresponds to a marginal use of a particular process; this represents that a

\footnotetext{
15 When considering other, more specific kinds of imagination acts, it could be necessary to add additional vertex to the rhombus. For instance, a vertex accounting for actions, in the case of pretense, or another one accounting for emotions, in the case of empathy.
} 
certain kind of imagination acts does not rely at all, or very little, on that specific process, and that its uses are almost negligible in the overall act.

2. The second measure, placed at the center of the line, corresponds to a standard use of the process; it is used regularly during the act of imagination and it adds useful information to the imaginary world that is being entertained.

3. The third measure, placed at the vertex of the outer rhombus, corresponds to a high use of the process; it is considered to be crucial in order to reach the goal the agent had in mind when engaging in such act of imagination.

Therefore, when evaluating a specific kind of acts of imagination using this tool, we draw a rhomboidal shape, called the inner rhombus and embedded within the outer rhombus, and which corresponds to a sort of blue-print representing the way this kind of imagination acts use each one of the processes. The Rhombus of Imagination is as shown in Fig. 9.

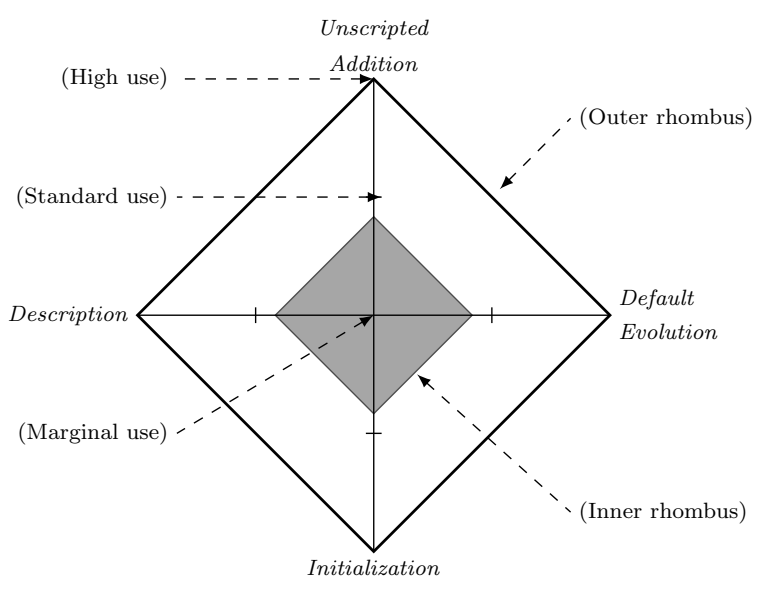

Fig. 9: The Rhombus of Imagination.

In order to analyze different kinds of imagination acts, we must consider acts of imagination in a general way; that is, how much do imagination acts of a certain kind usually rely on each process. Obviously, different instances of acts of imagination use each one of the different processes in different ways, but what the Rhombus of Imagination aims to capture is how relevant they typically are, for that particular kind of imagination acts. In order to show how the Rhombus works, let's consider three different cases: hypothetical reasoning, story-telling, and engaging in a preexisting fiction. 


\subsection{Hypothetical Reasoning}

Hypothetical reasoning is used to guide our actions and enhance our knowledge and beliefs about the real world. In this setting, therefore, imagination is highly constrained by reality-oriented rules and facts, as argued by authors such as Williamson (2016), Balcerak-Jackson (2016), or Spaulding (2016). Coming up with unexpected plot twists, or evolving the scenario in ways it would seldom do, falls outside the point of these kind of imagination acts. When defining the Rhombus of Imagination for Hypothetical Reasoning, we identify the following patterns:

1. Hypothetical reasoning requires the initial details of the imaginary scenario to be clearly set in order to ensure that the unfolding of further details will be reliable. We consider that hypothetical reasoning makes a high use of the Initialization process.

2. When entertaining an hypothetical scenario, one can be concerned with how a static scenario would be. For instance, when imagining how the staging of a theater play could be, or how one could decorate a room for a surprise birthday party, hypothetical reasoning allows us to foresee how a scenario would look like, rather than what would happen in there. In this sense, hypothetical reasoning often makes a high use of the Description process as well.

3. One of the most common uses of hypothetical reasoning usually involves predicting which events would take place in an alternative scenario, if it was real. Thus, it also makes a high use of the Default Evolution process.

4. In hypothetical reasoning we are not interested in voluntarily adding facts to the hypothetical scenario that would hardly follow, if it was real. Therefore, hypothetical reasoning makes a marginal use of the Unscripted Addition step.

Following the previous considerations, the Rhombus of Hypothetical Reasoning is as shown in Fig. 10. As it can be seen, this sort of imagination acts make extensive use of three of the processes identified in the proposed Common Frame for Imagination Acts, but it neglects the Unscripted Additions, which is consistent with the fact that their goal is to provide trustworthy information based on our knowledge and beliefs about the real world.

\subsection{Story-Telling}

Things change dramatically, though, when considering other kinds of imagination acts. When engaging in, say, story-telling, theater improvisation, or pretense play, the aim of such imaginings often goes beyond developing imaginary scenarios in the way they would normally do, if they were real. Instead, we usually expect for unexpected, or amusing things to happen in those fictional scenarios. Although reality-oriented rules and facts ${ }^{16}$ should also be present

\footnotetext{
16 In paradigmatic cases of fiction, such as medieval fantasy stories, this set of realityoriented rules and facts is not only formed by those rules the agent believes about the
} 


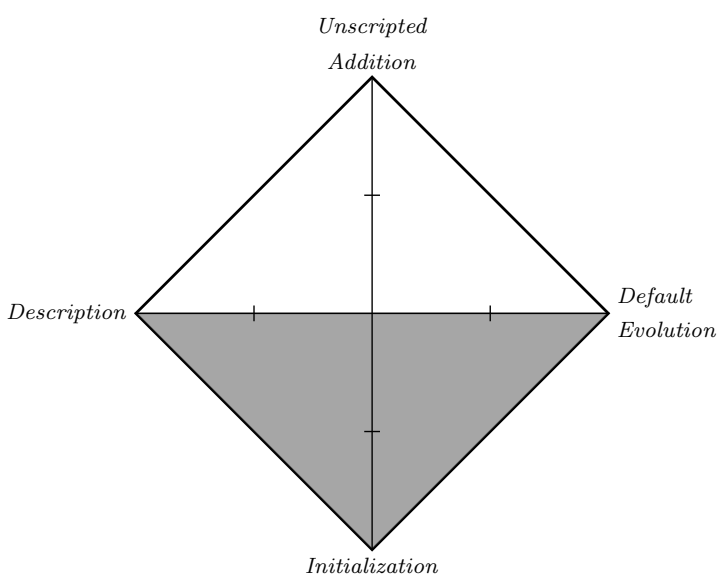

Fig. 10: The Rhombus of Hypothetical Reasoning.

in order to build a common grounds in which the imagining takes place, once the scenario has been set we need something else to make these imaginative episodes genuinely interesting.

Therefore, we need imagination to come up with unexpected twists and funny, or tragic situations that would not naturally follow from the imagined scenario. The Rhombus of Story-Telling can be defined by the following patterns:

1. As argued in the hypothetical reasoning case, every act of imagination makes use of this step: after all, it is through it that the imaginary scenario is created. However, the initial conditions describing an imaginary scenario are not usually as important when engaging in story-telling as they are in hypothetical reasoning; at least, the consequences of initiating the scenario with a less strict set of initial premises are not as severe in this case. Thus, we consider that story-telling makes a standard use of the Initialization step.

2. Embellishing the initial scenario with details regarding how the scenario looks like is usual in this kind of imaginings. This process is responsible for describing what elements are there in a pretense tea-party, for instance. Therefore, this kind of imagination acts makes a standard use of the Description step as well.

3. Similarly, allowing the imaginary scenario to evolve in ways it would typically do is something usual in this case. The Default Evolution is not what characterizes this kind of imagination acts, but nevertheless it is present, and we typically make use of it throughout the imaginative scenario. Thus,

real, actual world, but also by those rules that usually apply to the fictional setting being depicted. This latter kinds of rules correspond to Genre Truths, as identified in Funkhouser and Spaulding (2009). Additionally, but still related to this kind of paradigmatic rules, Lewis (1978) offers an account on how to assess truth in fiction. 
this kind of imagination acts make a standard use of the Default Evolution step.

4. Good stories usually tell us something unexpected, funny, interesting, mysterious, or tragic. These unexpected twists are seldom considered "usual ways" a scenario would typically develop, and so they fall under the scope of the Unscripted Additions. This is the most characteristic part of this kind of imagination acts, and thus we assign a high use to the Unscripted Additions process.

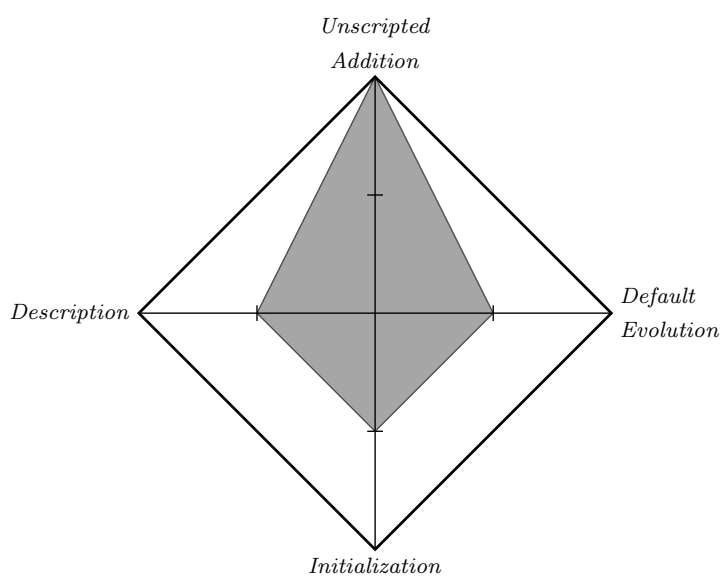

Fig. 11: The Rhombus of Story-Telling.

The Rhombus of Story-Telling is as shown in Fig. 11. As we can see, and when comparing it to the rhombus in Fig. 10, they are dramatically different. The Rhombus of Story-Telling has a highly-pointed vertex towards the Unscripted Additions, whereas the Rhombus of Hypothetical Reasoning is completely flat on that side. Conversely, the latter makes a high use of the processes that rely on reality-oriented rules and scripts, whereas their use is lower in the Rhombus of Story-Telling.

\subsection{Engaging in a Preexisting Fiction}

There is yet another kind of imagination act we want to represent: engaging in an already existing fiction - say, by reading a novel, or by listening to someone telling a story. The interest beyond this kind of imagination acts lies in the fact that, while being related to story-telling as well, the role of the agent changes dramatically, depending on whether the agent is the one telling the story (or writing the book), or listening to it (or reading it). Whereas in story-telling the agent is the one who creates the story, and thus has to use plenty of her 
own imaginative resources to tailor it, when the agent becomes the "receiver" of an existing fiction her imaginative resources are highly guided by the story being told.

The patterns followed when engaging in a fiction, and that determine the shape of the Rhombus of Imagination for this kind of imagination acts, are as follows:

1. As it happens when considering hypothetical reasoning, this step is crucial when engaging in a preexisting fiction in order to properly follow the story afterwards. The premises describing the scenarios involved in the story are being fed into our imagination in a way that allows us to imagine and follow what happens in them. Therefore, this step is of great importance in this sort of imagination acts, and we consider that engaging in preexisting fictions involves a high use of the Initialization step.

2. Although many details regarding how the scenario looks like can be given by the story-teller, many other details are left unspecified. All these unspecified details, although being prompted by a certain set of initial details, fall within the second process identified in our Common Frame. Thus, we assign a standard use of the Description step to this kind of imagination acts.

3. A similar thing happens regarding the way an imaginary scenario would usually evolve. While listening to a story, we do not imagine the characters doing what we think they would usually do, but rather doing what the story tells us they do. We do, however, assume certain "off-story" actions when prompted with certain clues, or when there is a time lapse in the story. Due to this, this kind of imagination acts make a standard use of the Default Evolution step as well.

4. Regarding the last process, its use is practically negligible in this kind of imagination acts. When listening to, or reading a story, we do not imagine that the characters do something different as what we are being told, nor we imagine that the story takes place somewhere else. Therefore, engaging in a preexisting fiction makes a marginal use of the Unscripted Additions.

The Rhombus of Engaging in a Preexisting Fiction is represented in Fig. 12. There are some interesting things we can say when comparing this Rhombus to the previous ones; in particular, neither the Rhombus of Hypothetical Reasoning (in Fig. 10), nor this one, make any use of the Unscripted Additions. The reasons for this are that both uses of imagination are constrained by a very specific set of rules and scripts, and they both aim to develop the imaginary scenario (be it reality-oriented or not) by following these rules and scripts. When engaging in hypothetical reasoning, we draw from our own knowledge and beliefs about reality in order to unfold the imaginary scenario; when engaging in a fiction, we are given the way an imaginary scenario advances, and we only make a light use of our knowledge and belief to embellish it. However, we do not intentionally deviate the scenario from what these rules and scripts tell us in neither case. Regarding the Rhombus of Story-Telling (in Fig. 11), their differences result from a "role-switching": being the one who comes 
up with the story requires a quite different use of one's imaginative resources than being the one who listens to it. This is represented by the fact that both Rhombuses have little in common, specially regarding the Initialization and the Unscripted Additions.

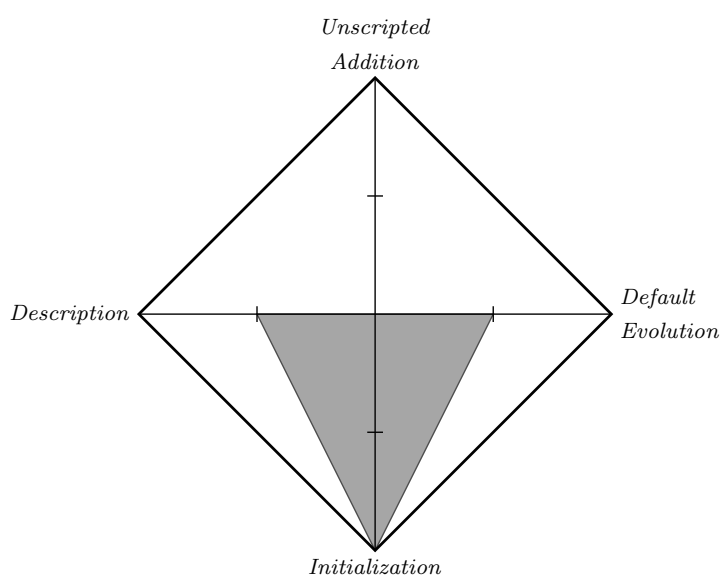

Fig. 12: The Rhombus of Engaging in a Preexisting Fiction.

4.4 The Rhombus as an Analysis Tool for Imagination Acts

By exploring the different ways in which imagination can be put to use, this Rhombus can be useful to further refine the distinction between closely-related acts, like "supposing", "assuming", "conceiving", or "imagining".

Even though we have chosen to treat them all as forms of a more general notion of "imagination" in order to focus on the content of such mental representations, and as we said at the beginning of this manuscript, some authors distinguish those acts. Weinberg and Meskin (2006), or Balcerak-Jackson (2016), for instance, argue that supposition is more commonly used during hypothetical reasoning, whereas imagination is more commonly used when engaging in fictions. We argue that, by being able to highlight the dynamic mechanisms more commonly used in certain imagination acts, the Rhombus of Imagination provides a clearer representation of intuitive differences between those. By representing the procedural signature of imagination in, say, hypothetical reasoning, the Rhombus captures what could be seen as the signature of "supposition", according to the previously cited works.

Furthermore, and as we point out on a footnote at the beginning of Section 4, the Rhombus could be extended to account for other mechanisms, such as the involvement of actual mental imagery. In that case, the Rhombus could be used to highlight how, according to certain authors, "imagining" does involve 
mental imagery, whereas "conceiving", or "supposing", do not. Even though this falls outside the scope of the present work, we claim that the Rhombus can be used to further refine and identify the differences between such acts in a more concrete way.

Alternatively, and moving beyond the debate about whether and how distinct those acts are, the Rhombus offers an alternative approach towards classifying acts of imagination. By paying attention not to the particular underlying act, but rather to the context in which a certain imagination episode is carried out, the Rhombus allows to distinguish and classify different "modes" of imagining, according to their procedural structure. Under this view, acts of imagination can be distinguished solely on their procedural signature, which determines which, and how much, they typically use one or another mechanism. Different kinds of imagination acts would be distinguished, under this interpretation, not through different mental states, but rather through the different contexts in which they are applied and their Rhombus signature.

\section{Conclusions and Future Work}

While reviewing certain theories of the dynamics of imagination in Section 2, we have seen how, although they all share an underlying structure, they are not detailed enough to be used as the underlying setting for a formal account of how imaginary worlds are created and elaborated. In particular, the way they define the reality-oriented development of imaginary scenarios embeds two processes that should be distinguished. The reasons behind such distinction are grounded both on the way actions had been previously represented in other logical systems, as well as on the distinct uses those processes have in different kinds of imagination acts. The Common Frame for Imagination Acts identifies four different processes occurring in acts of imagination and characterizes how they work in detail. We claim that those processes are better suited for a formal and computational study of the dynamics of imagination acts, in particular regarding how imaginary worlds are created and developed. We support our claim by providing a sketch of how a commonly known thought experiment could be formally represented using our framework.

Then, we define the Rhombus of Imagination as a visual tool specially suited to represent different kinds of imagination acts according to their procedural structure, and we show how the Rhombus works in some examples. Furthermore, we suggest how the Rhombus could be used to further understand the similarities and differences of distinct uses of imagination. This could either be done by focusing on the context in which imagination is applied, or by looking at specific kinds of imagination acts, such as supposing, or conceiving, to mention a few.

The Common Frame for Imagination Acts opens up the possibility of capturing the dynamics of imagination acts using formal tools and computational simulations. A natural next step, therefore, is to design and implement the intuitions captured by the Common Frame into formal systems and further 
explore how our proposal can provide interesting insights to the study of imagination acts. Although we have sketched our proposal with a classical possibleworld setting in mind, considering a paraconsistent setting such as the one used in Berto (2017), or Priest (2016), could lead to interesting and versatile systems for imagination. Additionally, being able to capture such dynamics in a computer-based simulation would be useful for the formal study of certain reasoning mechanisms that rely heavily on imaginary scenarios, such as thought experiments.

Furthermore, and after seeing how the Rhombus of Imagination can indeed be used as a valuable tool for identifying dynamic properties of imagination, we want to perform a comprehensive analysis of the different kinds of imagination acts that appear in the relevant literature in order to identify their procedural differences. We believe that the analysis of their dynamics will provide valuable insights regarding how they work, and how distinct kinds of imagination resemble and differ from other ones.

\section{References}

Balcerak-Jackson M (2016) On the epistemic value of imagining, supposing and conceiving. In: Kind A, Kung P (eds) Knowledge Through Imagination, OUP Oxford, chap 1, pp 41-60

Berto F (2014) On conceiving the inconsistent. In: Proceedings of the Aristotelian Society (Hardback), Wiley Online Library, vol 114, 1.1, pp 103-121

Berto F (2017) Impossible worlds and the logic of imagination. Erkenntnis 82(6):1277-1297

Berto F (2018) Aboutness in imagination. Philosophical Studies 175(8):18711886

Blackburn P, De Rijke M, Venema Y (2010) Modal Logic. Cambridge University Press

Costa-Leite A (2011) Logical properties of imagination. Abstracta 6(1):103116

Currie G, Ravenscroft I (2002) Recreative Minds: Imagination in Philosophy and Psychology. Oxford University Press

Funkhouser E, Spaulding S (2009) Imagination and other scripts. Philosophical Studies 143(3):291-314

Giordani A (2019) Axiomatizing the logic of imagination. Studia Logica 107(4):639-657

Harel D (1984) Dynamic logic. In: Gabbay DM, Guenthner F (eds) Handbook of Philosophical Logic, volume II: Extensions of Classical Logic, D. Reidel Publishing Company, pp 497-604

Hodges W (2001) Logic: An Introduction to Elementary Logic. Penguin Books

Kind A (2016a) Desire-like imagination. In: Kind A (ed) The Routledge Handbook of Philosophy of Imagination, Routledge, chap 12, pp 163-176

Kind A (ed) (2016b) The Routledge Handbook of Philosophy of Imagination. Routledge, Taylor \& Francis Group 
Kind A, Kung Pe (2016) Knowledge Through Imagination. OUP Oxford

Langland-Hassan P (2016) On choosing what to imagine. In: Kind A, Kung P (eds) Knowledge Through Imagination, OUP Oxford, chap 2, pp 61-84

Lewis D (1973) Counterfactuals. Blackwell Publishing

Lewis D (1978) Truth in fiction. American Philosophical Quarterly 15(1):37-46

Nanay B (2016) The role of imagination in decision-making. Mind and Language 31(1):126-142

Nichols S (2006) The architecture of the imagination: New essays on pretence, possibility, and fiction. Oxford University Press

Nichols S, Stich S (2000) A cognitive theory of pretense. Cognition 74(2):115147

Nichols S, Stich SP (2003) Mindreading: An integrated account of pretence, self-awareness, and understanding other minds. Clarendon Press/Oxford University Press

Niiniluoto I (1985) Imagination and fiction. Journal of Semantics 4(3):209-222

Priest G (2016) Thinking the impossible. Philosophical Studies 173(10):26492662

Rucińska Z (2016) What guides pretence? towards the interactive and the narrative approaches. Phenomenology and the Cognitive Sciences 15(1):117133

Shirazi A, Amir E (2007) Probabilistic modal logic. In: Holte R, Howe A (eds) Proceedings of the 22nd AAAI Conference on Artificial Intelligence, pp 489-495

Sinhababu N (2016) Imagination and belief. In: Kind A (ed) The Routledge Handbook of Philosophy of Imagination, Routledge, chap 8, pp 111-123

Sorensen RA (1992) Thought Experiments. Oxford University Press

Spaulding S (2016) Imagination through knowledge. In: Kind A, Kung P (eds) Knowledge Through Imagination, OUP Oxford, pp 207-226

Van Leeuwen N (2013) The meanings of "imagine" part i: Constructive imagination. Philosophy Compass 8(3):220-230

Velazquez Quesada FR (2011) Small steps in dynamics of information. Institute for Logic, Language and Computation

Walton KL (1990) Mimesis as make-believe: On the foundations of the representational arts. Harvard University Press

Wansing H (2017) Remarks on the logic of imagination. a step towards understanding doxastic control through imagination. Synthese 194(8):2843-2861

Weinberg JM, Meskin A (2006) Puzzling over the imagination: Philosophical problems, architectural solutions. In: Nichols S (ed) The architecture of the imagination: New essays on pretence, possibility, and fiction, Oxford University Press, chap 10, pp 175-202

Williamson T (2016) Knowing by imagining. In: Kind A, Kung P (eds) Knowledge Through Imagination, OUP Oxford, chap 4, pp 113-123 